\title{
Sodium Alginate Intercalated Zinc-Containing Layered Double Hydroxides for the Catalytic $\alpha$-Pinene Oxidation
}

\author{
HOU YanChun, CHEN ChunXia and CHEN Chen \\ College of Science, Northeast Forestry University, Harbin, P. R. China \\ Correspondence should be addressed to: CHEN ChunXia; ccx1759@163.com
}

Received date: 22 February 2014; Accepted date: 30 April 2014, Published date: 12 September 2014

Academic editor: Sampak Samanta

Copyright (C) 2014. HOU YanChun, CHEN ChunXia and CHEN Chen. Distributed under Creative Commons CC-BY 3.0

\begin{abstract}
In this paper, the sodium alginate (SA) intercalated into a series of binary $\mathrm{Mg}-\mathrm{Al} 、 \mathrm{Cu}-\mathrm{Al}, \mathrm{Zn}-\mathrm{Al}$ flaky layered double hydroxides (FLDH/SA) were fleetly prepared by the technology of the microwave-crystallization and low saturated state of coprecipitation. The purposed sample was characterized by XRD, IR and SEM. The result shows that the sample synthesized Zn-FLDH/SA with the flexible slice has the structure of LDHs, indicating the preparations are successful under microwave irradiation. The three types of CFLDH/SA were the calcined products of the three FLDH/SA under $673 \mathrm{~K}$ for $4 \mathrm{~h}$, which were then applied to the catalytic $\alpha$-pinene oxidation using 30 wt $\%$ hydrogen peroxide as oxidant. The effects of reaction time, catalyst type, reaction medium and so on were also investigated in details. The results show the Zn-CFLDH/SA is the best catalyst, $\alpha$-pinene conversion and the selectivity of $\alpha$-pinene epoxide, verbenol, verbenone can reach $69.6 \%$ and $29.1 \%, 39.6 \%, 12.0 \%$, respectively, under optimized reaction conditions of acetone: water $=1: 1$ as reaction solvent, catalyst concentration of $0.431 \mathrm{~g} / \mathrm{L}$, reaction temperature $60^{\circ} \mathrm{C}$ and reaction time 3 hours, Zn-CFLDH/SA as catalyst.
\end{abstract}

Keyword: Sodium alginate; Zinc-containing layered double hydroxides; Green catalyst; $\alpha$-Pinene oxidation

\section{Introduction}

The innovation of catalytic materials as powerful tools for the transformation of chemical raw materials in the forest is greatly significant in the production and application of forestry chemicals. As the main component of turpentine oil, $\alpha$-pinene not only is a kind of

Cite this Article as: HOU YanChun, CHEN ChunXia and CHEN Chen (2014), "Sodium Alginate Intercalated Zinc-Containing Layered Double Hydroxides for the Catalytic $\alpha$-Pinene Oxidation", Journal of Research \& Developments in Chemistry, Vol 2014 (2014), Article ID 431101, DOI: 10.5171/2014.431101 
renewable starting material for producing a wide variety of valuable products, but also a readily available and inexpensive of natural resources. Specifically, its oxidation products (see Scheme 1) such as verbenol, verbenone and $\alpha$-pinene epoxide are of high practical importance as flavor chemicals and key intermediates for the manufacture of various fine chemicals, as Maksimchuk et al (2005) mentioned, including medicine intermediates, spice additives, fragrances, and agrochemicals, citral, menthol, taxol etc.

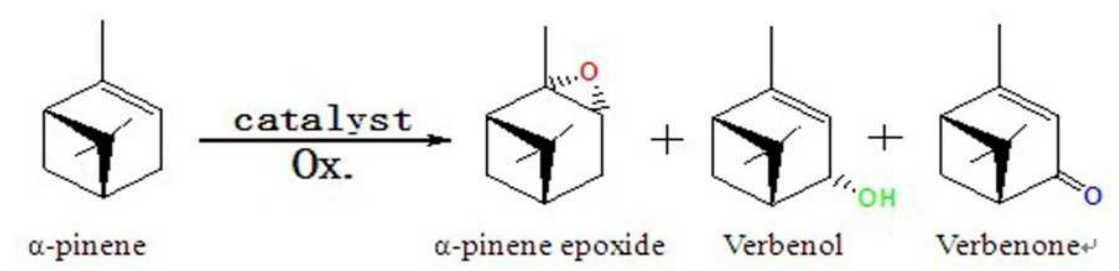

Scheme1 The reaction of $\alpha$-pinene oxidation

Much effort in the $\alpha$-pinene oxidation reaction has been mainly devoted to the survey of the new recyclable catalytic system with the environmentally friendly oxidants. Hydrogen peroxide is as the oxidant, which has a large number of advantages including (a) it simplifies greatly the work-up to avoid the separation of the carboxylic acid as by-product, (b) it produces a lower amount of water as the only by-product and is environmentally friendly, (c) it is commercially available, inexpensive, and easy to handle, (d) it contains a higher concentration of active oxygen (30 wt\%) than all other oxidants except molecular oxygen, therefore it can improve process economics. On the other hand, hydrogen peroxide is kinetically more inert with respect to organic peracids and its direct use, as the oxidant requires a suitable catalyst for sufficient activity and excellent selectivity.

The main three products of $\alpha$-pinene oxidation are $\alpha$-pinene epoxide, verbenol and verbenone. In addition, the existence of other byproducts such as camphene and sobrerol etc, which cannot be avoided due to the active allyl-bit and its special double bonds, therefore, the catalyst plays a key role between the two competing reactions. Different catalysts lead to different conversions and generating the main three kinds of product ratio being different, the property of a catalyst decides the consequence. In a research study by Tzialla (2010), the use of a number of different catalysts has dramatically revolutionized oxidation processes with hydrogen peroxide. In recent years, Qi (2010) and Patil (2001) have focused on the development of heterogeneous catalysts that accord with green chemistry. Manganese and cobalt (II)-containing zeolite have emerged as the major breakthrough in the alkenes epoxidation using $\mathrm{H}_{2} \mathrm{O}_{2}$ as oxidant. Chakrabarty (2004) mentioned tetrameric cobalt (III) complexes, Timofeeva (2012) mentioned vanadium-based catalysts on mesoporous supports, and Nagendrappa's (2011) clay-based catalysts have been successfully used for the $\alpha$-pinene oxidation. Layered double hydroxides (LDHs), known as hydrotalcite-like compounds (HTLc), have been widely applied in various organic reactions, such as in the research study by Kristina (2014) and Tatsumi (1993), with advantages such as effective activity, high selectivity, excellent metal dispersion, less waste production, and an improved recuperation of immobilized catalysts. Various catalysts based on LDHs structures are used in alkene oxidation reactions. Aramendia (2000) discussed the catalytic properties of different LDHs in the epoxidation of limonene to limonene oxide, and Kaneda (1995) uses molecular oxygen and aldehydes as a combination oxidant of several ketones. Through high activity, selectivity, and chiral induction, this system also has several disadvantages, including the fact that the flow rate of oxygen cannot be easily controlled and it is difficult to realize industrialization. 
Mandal (2012) mentioned that, alginates are important, naturally occurring biopolymers and the block copolymers of uronic acids, a structural component of an algal cell wall, has been widely used for biomolecule immobilization and is a potent metal chelator. Sodium alginate can be exchanged by metal cations and then be coordinated with the $-\mathrm{OH}$ of the carboxylate groups to generate the ion-cross-linked alginates. They have been used in several applications. In the research study by Shi (2012) and Monakhova (2012), organic-inorganic composite materials containing various salts of alginic acid were found to be useful for application in catalysis.

Therefore, in the present work, we synthesized sodium alginate intercalated the three types of flaky layered double hydroxides (FLDH/SA) under the microwave irradiation. At the same time, the as-synthesized samples were applied in the catalytic $\alpha$-pinene oxidation with the $\mathrm{H}_{2} \mathrm{O}_{2}$ as oxidant. Factors affecting the reaction with $\mathrm{Zn}-\mathrm{CFLDH} / \mathrm{SA}$ as the catalyst, such as reaction medium, and reaction time were also investigated in details.

\section{Experimental}

\section{Materials}

All chemicals were analytical grade without further purification. Zinc nitrate, copper nitrate, magnesium nitrate, aluminium nitrate, sodium hydroxide and sodium alginate (SA) were purchased from Tian Jin Kemiou Fine Chemical Reagent. Acetone, ethanol, $\mathrm{CH}_{2} \mathrm{Cl}_{2}$, $\mathrm{CHCl}_{3}$, methanol and n-decane were offered from Tianjin Heowns Biochemical Technology Chemical Industry. Standard samples including $\alpha$-pinene, $\alpha$-pinene epoxide, verbenol and verbenone were offered from Aladdin Reagent.

\section{Preparation of Catalysts}

An $80 \mathrm{ml}$ aqueous solution containing $60 \mathrm{ml}$ bivalent metal nitrates $\left(\mathrm{Mg}\left(\mathrm{NO}_{3}\right)_{2} 、 \mathrm{Cu}\left(\mathrm{NO}_{3}\right)_{2}\right.$ or $\mathrm{Zn}\left(\mathrm{NO}_{3}\right)_{2}$ and $20 \mathrm{ml}$ trivalent metal nitrates $\left(\mathrm{Al}\left(\mathrm{NO}_{3}\right)_{3}\right)$, with another $140 \mathrm{ml}$ solution, which containing $1 \mathrm{~mol} \cdot \mathrm{L}^{-1} \mathrm{NaOH}$ and 0.01 $\mathrm{mol} \cdot \mathrm{L}^{-1}$ SA solution were simultaneously titrated into a three necked round-bottom flask under vigorous stirring at $70{ }^{\circ} \mathrm{C}$. The titration rates of the solutions were controlled in order to maintain the $\mathrm{pH}$ value of the mixture solution at about 8-9. After the titration and one hour's vigorous stirring, a heavy precursor gel was obtained, and then crystallized at $70{ }^{\circ} \mathrm{C}$ for 15 minutes under microwave irradiation (Midea KD23B-DA, at max power $1300 \mathrm{~W}$ and a frequency of 2.45 $\mathrm{GHz}$ ). The precipitate was filtered, washed to pH 7 and dried to obtain the three types FLDHs/SA. The purposed sample was calcined under $673 \mathrm{~K}$ for $4 \mathrm{~h}$ to obtain the CFLDH/SA. $\mathrm{Zn}$-FLDH has the same synthetic procedure as above, just without the intercalation agent SA.

\section{Characterization of Catalysts}

X-ray powder diffraction (XRD) data were characterized on a Japan Rigaku D/MAX 2200VPC. Data were collected at the rate of $4 \%$ min and in the range of 5-80․ The Fourier transform infrared (FT-IR) spectra were collected using a Nicolet FT-IR 360 spectrometer $\left(\mathrm{KBr}\right.$ pellet method, $4 \mathrm{~cm}^{-1}$ resolution) in the range of $400-4000 \mathrm{~cm}^{-1}$. The morphology of sample was observed by FEI-Quanta 200 scanning electron microscope (SEM) with a field emission of $30 \mathrm{Kv}$.

\section{Pinene Oxidation}

Oxidation of $\alpha$-pinene over the prepared catalyst with $30 \mathrm{wt} \%$ hydrogen peroxide was carried out in a $15 \mathrm{ml}$ sealed reaction tube (with stir). In a typical experiment, reaction mixture containing known amounts of $\alpha$-pinene, catalyst and specific solvent $(2 \mathrm{ml}$ ). The reaction products were analyzed by gas chromatography (Agilent, Model 6820) equipped with HP-5 capillary column $30 \mathrm{~m}$ long and $0.25 \mathrm{~mm}$ internal diameter and a flame ionization detector (FID). Nitrogen was used as a carrier gas with injection port temperature of $593 \mathrm{~K}$ and programmed ramping of column temperature from $333 \mathrm{~K}$ to $533 \mathrm{~K}$. The $\mathrm{n}$-decane was used as an internal standard for calculation.

\section{Results and Discussion}

\section{Characterization of Catalysts}

Fig.1 illustrates the powder X-ray diffraction (XRD) patterns of Zn-FLDH/SA and Zn-CFLDH/SA. A gives several typical 
reflections at $2 \theta=10.2,20.0,34.4,61.1$, which Rives (2003) mentioned in his paper and confirmed that no significant structural variations occurred after SA intercalated as compared to the original typical reflections, and also shows that it is highly crystalline material. $\mathrm{B}$ gives some typical reflections at $2 \theta=32.1,36.2,47.1,57.3,63.2,66.5$, which are almost identical to the typical pattern characteristic of $\mathrm{ZnO}$ as Shi (2013) reported, while the package peaks between $34.22 \sim 37.5$ due to the amorphous peak of $\mathrm{Al}_{2} \mathrm{O}_{3}$ as Constantino (1994) mentioned. It can be concluded that the Zn-CFLDH/SA is mostly in the form of metal oxide.

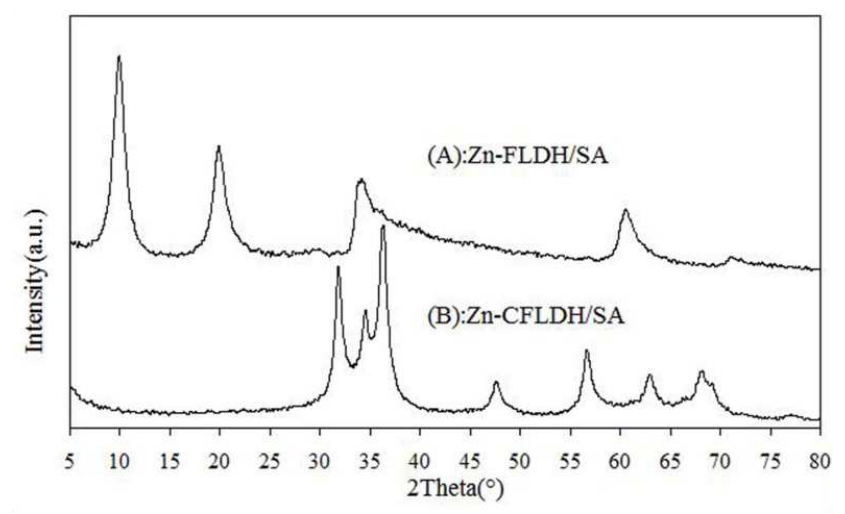

Fig.1 X-ray diffraction patterns of Zn-FLDH/SA (A) and Zn-CFLDH/SA (B)

In Fig.2, it can be observed that both samples show strong bands at $3456 \mathrm{~cm}^{-1}$ and 1384 $\mathrm{cm}^{-1}$, as well as a group of other small bands below $1200 \mathrm{~cm}^{-1}$ in the FT-IR spectrum (Fig.2), which Chen (2005) mentioned, and confirmed that the two samples exhibit the structure of hydrotalcite-like compounds. The band centered at around $3456 \mathrm{~cm}^{-1}$ is due to the $v-\mathrm{OH}$ stretching mode of hydrogen-bonded hydroxyl groups and water molecules. The shaper band at $1630 \mathrm{~cm}^{-1}$ ( Fig2.B ) is attributed to $\mathrm{C}=\mathrm{O}$ stretching vibrations of Zn-FLDH/SA besides $\mathrm{M}-\mathrm{OH}$ vibration in the brucite-like layers of the two kinds of $\mathrm{Zn}-\mathrm{FLDH}$, indicating the presence of the $\left[\mathrm{C}_{5} \mathrm{H}_{7} \mathrm{O}_{4} \mathrm{COO}\right]^{-}$ ion within the layered double hydroxide. All the characteristic peaks prove that the SA was intercalated into Zn-FLDH successfully.

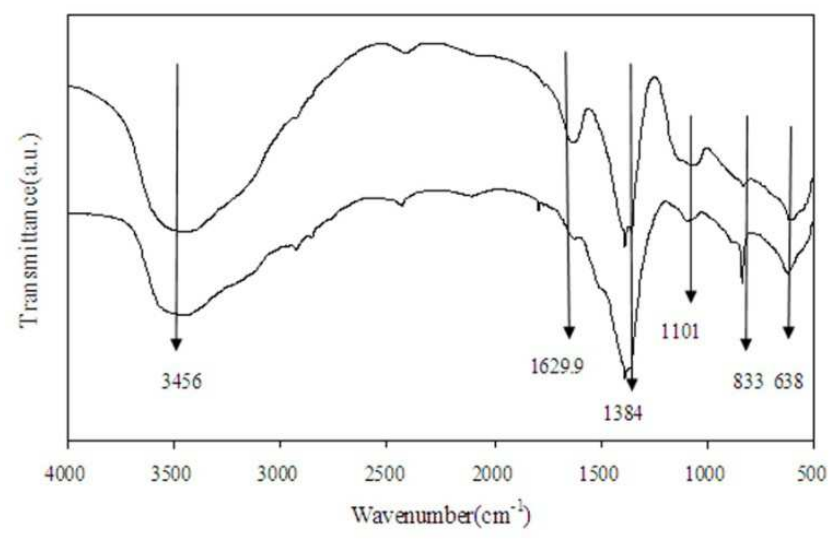

Fig.2 FT-IR spectra of Zn-FLDH/SA (A) and Zn-CFLDH/SA (B) 
Fig.3 A and B illustrates the SEM images of Zn-FLDH/SA and Zn-CFLDH/SA, respectively. The A image shows that the sheet particles of Zn-FLDH/SA form petal-like structures with many cavities, and this leads to the hierarchical structure stacking of LDHs nanoflakes according Zhi's report (2010). This compound consists of interleaved flexible curled nanosheets that are $10-20 \mathrm{~nm}$ thick and 200-500 $\mathrm{nm}$ long. This is possibly because in the intercalation reaction of SA, its large ion results in the expansion of LDHs, and the interlayer spacing increases gradually along with the insertion of SA. When the interlayer spacing becomes large enough such that the interaction force between the layers completely disappears, the LDHs appears as monolayer, which then self-assembles into the Zn-FLDH diaphragm in the saturated solution of SA and forms Zn-FLDH/SA nanoflakes. $\mathrm{Zn}-\mathrm{CFLDH} / \mathrm{SA}$ is maintaining the original morphology, apparently, which has flake-like crystals stacked over one another to form layers. But during the calcinations, the water in the laminate and the anion SA-intercalation peeled out from the Zn-CFLDH/SA structure, these lead to more cavities.

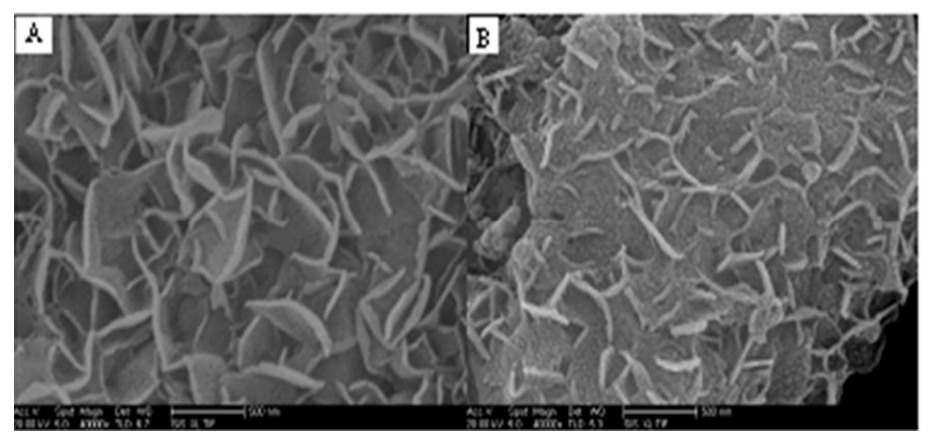

Fig.3 SEM images of Zn- FLDH/SA (A) and Zn-CFLDH/SA (B)

$\alpha$-Pinene Oxidation

\section{Effect of Catalyst Type}

LDHs are efficient catalysts for alkene oxidation carried out, which have been reported by Ray (2011) and Mitran (2012) in previous literature. However, there are still few reports about $\alpha$-pinene oxidation using hydrogen peroxide as oxidant catalyzed by LDHs. In the present work, the catalytic performances of prepared catalysts were investigated in the $\alpha$-pinene oxidation; the results are summarized in Table 1 . From Table 1, it can be easily found that, compared with FLDH/SA, the CFLDH/SA have higher activity. When we just observe the activity of Zn-containing FLDH, the activity order is Zn-CFLDH/SA > Zn-FLDH/SA > Zn-CFLDH > $\mathrm{Zn}-\mathrm{FLDH}$, the difference of conversion rate is evident. Therefore, it can be concluded that the SA in Zn-FLDH/SA plays a key role, as Bhattacharjee (2004) mentioned the gallery height may be increased sufficiently to allow the oxidation of larger dimension organic compounds, and Zn-CFLDH/SA has the same number of active centers and more cavities. Moreover, it can be observed that the main product is verbenol, verbenone and $\alpha$-pinene epoxide. Additionally, from the above-obtained results in Table 1 , it can be observed that the Zn-CFLDHs/SA exhibits the higher conversion of $\alpha$-pinene and selectivity of verbenol, indicating that Zn-CFLDHs/SA is the best catalyst among all the synthesized catalysts in $\alpha$-pinene oxidation reaction. Therefore, the present work further investigates the catalytic performances of Zn-CFLDHs/SA in $\alpha$-pinene oxidation with various reaction parameters in details. 
Table 1: Catalytic properties of prepared catalyst in $\alpha$-pinene oxidation

\begin{tabular}{|l|l|l|l|l|l|}
\hline Catalyst type & $\mathbf{C \%}$ & $\mathbf{1 S \%}$ & $\mathbf{2 S \%}$ & $\mathbf{3 S \%}$ & other\% \\
\hline Zn-CFLDH & 42.1 & 33.5 & 18.9 & 33.5 & 14.1 \\
\hline Zn-FLDH & 39.3 & 40.9 & 18.9 & 33.6 & 6.6 \\
\hline Zn-CFLDH/SA & 69.6 & 29.1 & 39.6 & 12 & 19.3 \\
\hline Zn-FLDH/SA & 51.7 & 35.4 & 17.9 & 35.9 & 10.8 \\
\hline Mg-CFLDH/SA & 58.2 & 32.4 & 29.6 & 28 & 10 \\
\hline Mg-FLDH/SA & 48.8 & 30.2 & 28.8 & 23 & 18 \\
\hline Cu-CFLDH/SA & 55 & 31.6 & 26.7 & 29 & 12.7 \\
\hline Cu-FLDH/SA & 46.7 & 29.2 & 25.3 & 16.8 & 28.7 \\
\hline
\end{tabular}

Reaction conditions: $1 \mathrm{mmol} \alpha$-pinene, $1 \mathrm{mmol} \mathrm{H}_{2} \mathrm{O}_{2}, 10 \mathrm{mg}$ catalysts, $1 \mathrm{ml}$ acetone and $1 \mathrm{ml}$ distilled water, temperature $60^{\circ} \mathrm{C}$, time $180 \mathrm{~min}$.

C\%——conversion of $\alpha$-pinene, 1S\%——selectivity of $\alpha$-pinene epoxide

$2 \mathrm{~S} \%$ - - selectivity of verbenol, $3 \mathrm{~S} \%$ - selectivity of verbenone

\section{Effect of Reaction Medium}

In order to investigate the effect of the reaction medium on the $\alpha$-pinene oxidation catalyzed by $\mathrm{Zn}-\mathrm{CFLDH} / \mathrm{SA}$, the reaction was performed in various solvents such as water, acetone, mixtures thereof, $\mathrm{CH}_{2} \mathrm{Cl}_{2}$, methanol and $\mathrm{CHCl}_{3}$. The results are summarized in Table 2. From Table 2, it can be clearly seen that the Zn-CFLDH/SA-catalyzed $\alpha$-pinene oxidation gives the highest $\alpha$-pinene conversion in acetone: water $=1: 1$ as solvent, however, and has lower catalytic activity in anhydrous solvents. This is perhaps due to the following reasons: (1) $\alpha$-pinene is the insoluble organic matter in water, so the reaction of hydrogen peroxide and $\alpha$-pinene is two phases of liquid-liquid reaction; (2) adding the solid catalyst, the whole reaction system is three phases of liquid-liquid-solid reaction, acetone/water as the solvent, $\alpha$-pinene of organic material is easy to contact with acetone, Calcined Zn-CFLDH/SA catalyst is hydrophilic, the solvent can make $\alpha$-pinene with hydrogen peroxide for miscibility of liquid-liquid-solid system to the liquid-solid two phases, which can be beneficial for reaction. Hence, acetone/water is indicated to be the best alternative solvent for the $\alpha$-pinene oxidation. The result is in agreement with the previous measurements of Mandelli (2011), the catalytic process, maybe, is a dehydration reaction a small amount of water is advantageous for the reaction. It can not only inhibit the decomposition of $\mathrm{H}_{2} \mathrm{O}_{2}$ in the catalyst surface, but also inhibits the catalytic epoxidation products of ring-opening reactions and rearrangement reactions.

Moreover, peroxide activates hydroxyl groups on the surface of the crystal and reacts with $\alpha$-pinene; the catalyst can keep high activity either in acidic, neutral or alkaline.

Table 2: Effect of reaction medium on $\alpha$-pinene oxidation catalyzed by Zn-CFLDH/SA

\begin{tabular}{|l|l|l|l|l|l|}
\hline medium & $\mathbf{C} \%$ & $\mathbf{1 S} \%$ & $\mathbf{2 S} \%$ & $\mathbf{3 S} \%$ & other\% \\
\hline Acetone: $\mathrm{H}_{2} \mathrm{O}=1: 1$ & 69.6 & 29.1 & 39.6 & 12 & 19.3 \\
\hline $\mathrm{H}_{2} \mathrm{O}$ & 36.9 & 27.7 & 18.7 & 21.6 & 32 \\
\hline Alcohol & 33.1 & 15.7 & 39 & 40.2 & 5.1 \\
\hline
\end{tabular}




\begin{tabular}{|l|l|l|l|l|l|}
\hline $\mathrm{CH}_{2} \mathrm{Cl}_{2}$ & 31.1 & 24 & 24.7 & 19.6 & 31.7 \\
\hline Methanol & 27 & 51.4 & 23.7 & 24.9 & 27 \\
\hline Acetone & 26.8 & 31.8 & 13 & 17.8 & 37.4 \\
\hline $\mathrm{CHCl}_{3}$ & 23 & 34.8 & 18.6 & 28.5 & 18.1 \\
\hline
\end{tabular}

Reaction conditions: $1 \mathrm{mmol} \alpha$-pinene, $1 \mathrm{mmol} \mathrm{H}_{2} \mathrm{O}_{2}, 10 \mathrm{mg}$ catalysts ( $\mathrm{Zn}$-CFLDH/SA), $2 \mathrm{ml}$ solvent, temperature $60{ }^{\circ} \mathrm{C}$, time $180 \mathrm{~min}$.

\section{Effect of Reaction Time}

The effect of reaction time on the $\alpha$-pinene oxidation reaction catalyzed by Zn-CFLDH/SA was investigated. The results are shown in Fig.4, which reveals that the $\alpha$-pinene conversion increases drastically with an increasing reaction time at the beginning and then levels off at $70 \%$ after 3 hours, because of the reaction fulfillment. However, the selectivities of the three products show major change and this maybe depends on the specific formed cation evolving after protonation of the a-pinene molecule at the beginning of the reaction.

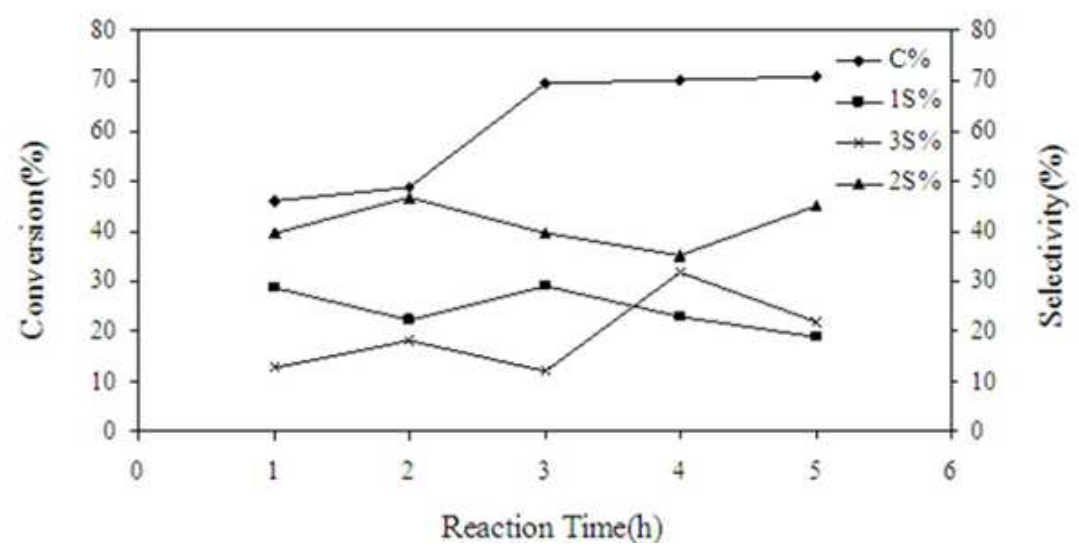

Fig. 4 Effect of reaction time

Reaction conditions: $1 \mathrm{mmol} \alpha$-pinene, $1 \mathrm{mmol}$ $\mathrm{H}_{2} \mathrm{O}_{2}, 10 \mathrm{mg}$ catalysts(Zn-CFLDH/SA), $1 \mathrm{ml}$ acetone and $1 \mathrm{ml}$ distilled water, temperature $60^{\circ} \mathrm{C}$.

\section{Conclusion}

FLDH/SA as catalyst precursors were prepared under microwave irradiation, which was calcined to catalyze the oxidation of $\alpha$-pinene with hydrogen peroxide as oxidant. The purposed sample was characterized by XRD, IR and SEM. The results show that the synthesized FLDH/SA with the flexible slice have the structure of $\mathrm{LDH}$, indicating the preparation is successful under microwave irradiation. The calcined product is uniform state. The catalytic activity order of the prepared catalyst for $\alpha$-pinene oxidation is Zn-CFLDH/SA >Zn-FLDH/SA

Zn-CFLDH $>$ Zn-FLDH, which imply SA plays an important role in the reaction and $\mathrm{Zn}-\mathrm{CFLDH} / \mathrm{SA}$ is the best catalyst for $\alpha$-pinene oxidation with $30 \mathrm{wt} \%$ hydrogen peroxide as oxidant. The conversion rate of $\alpha$-pinene can reach $69.6 \%$ under the reaction conditions of acetone: water $=1: 1$ as reaction solvent, Zn-CFLDH/SA catalyst concentration of 0.431 $\mathrm{g} / \mathrm{L}$ and reaction time of $3 \mathrm{~h}$.

\section{Acknowledgment}

The investigation was supported by the Fundamental Research Funds for the Central 
Universities (No. 2572014DB04) and the Specialized Research Fund for the Doctoral Program of Higher Educatio (No.20120062120012).

\section{References}

1. Aramendía, M. A, Borau, V. and Jiménez, C. (2000) "Epoxidation of limonene with layered-double hydroxides as catalysts." Stud Surf Sci Catal, 130:1667-1672.

2. Bhattacharjee, S. and Anderson, J. A. (2004) "Synthesis and characterization of novel chiral sulfonato- salen-manganese (III) complex in a zinc-aluminium LDH host ." Chem Commun, 5(1-2): 554-555.

3. Chakrabarty, R. and Das, B. K. (2004) "Epoxidation of $\alpha$-pinene catalysed by tetrameric cobalt (III) complexes." J Mol Catal A: Chem, 223(1-2):39-44.

4. Chen, C. X, Xu, C. H. and Feng, L. R . (2005) "Effect of Rare Earth Doping on the Catalytic Activity of Copper-Containing Hydrotalcites in Phenol Hydroxylation." Adv Synth Catal, 347(14): 1848-1854.

5. Constantino, V. R. L. and Pinnavaia, T. J. (1994) "Structure-reactivity relationships for basic catalysts derived from a $\mathrm{Mg}^{2+}-\mathrm{Al}^{3+}-\mathrm{CO}_{3}{ }^{2-}$ layered double hydroxide." Catal let, 23(3-4): 361-367.

6. Kaneda, K, Ueno, S. and Imanaka, T. (1995) "Catalysis of transition metal-functionalized hydrotalcites for the Baeyer-Villiger oxidation of ketones in the presence of molecular oxygen and benzaldehyde." J Mol Catal A: Chem, 102:135-138.

7. Kristina, K. R, Albinas, Ž. and Ričardas, T. (2014) "Preparation of Mg/Al layered double hydroxide (LDH) with structurally embedded molybdate ions and application as a catalyst for the synthesis of 2-adamantylidene(phenyl)amine Schiff base." Polyhedron, 68:340-345.

8. Maksimchuk, N. V, Melgunov, M. S. and Białoń J. M. (2005) " $\mathrm{H}_{2} \mathrm{O}_{2}$-based allylic oxidation of $\alpha$-pinene over different single site catalysts." J Catal, 2351(1): 175-183.
9. Mandal, S. and Patil, V. S. (2012)“Alginate and hydrotalcite-like anionic clay compositesystems:

Synthesis, characterization and application studies." Mayadevi S. Microporous Mesoporous Mater, 158:241-246.

10. Mandelli, D. and VanVliet, M. C. A. (2001) "Alumina-catalyzed alkene epoxidation with hydrogen peroxide." Appl Catal A, 219(1-2):209-213.

11. Mitran, G, Cacciaguerra, T. and Loridant, S. (2012) "Oxidative dehydrogenation of propane over cobalt-containing mixed oxides obtained from LDH precursors." Appl Catal A, 417:153-162.

12. Monakhova, Y, Agulhon, P. and Quignard, F. (2012) "New mixed lanthanum- and alkaline-earth cation-containing basic catalysts obtained by an alginate route."Catal Today, 189(1): 28-34.

13. Nagendrappa, G. (2011) "Organic synthesis using clay and clay-supported catalysts." Appl Clay Sci, 53(2):106-138.

14. Patil, M. V, Yadav, M. K. and Jasra, R. V. (2007) "Catalytic epoxidation of $\alpha$-pinene with molecular oxygen using cobalt(II)-exchanged zeolite Y-based heterogeneous catalysts." J Mol Catal A: Chem, 277(1-2):72-80.

15. Qi, B, Lu, X. H. and Zhou, D. (2010) "Catalytic epoxidation of alkenes with 30\% $\mathrm{H}_{2} \mathrm{O}_{2}$ over $\mathrm{Mn}^{2+}$-exchanged zeolites." J Mol Catal A: Chem, 322(1-2):73-79.

16. Ray, A, Rosair, G. M. and Pilet, G. (2011) "Preferential azido bridging regulating the structural aspects in cobalt(III) and copper(II)-Schiff base complexes: Syntheses, magnetostructural correlations and catalytic studies." Inorg Chim Acta, 375(1): 20-30.

17. Rives, V, Prieto, O. and Dubey, A. (2003) "Synergistic effect in the hydroxylation of phenol over CoNiAl ternary hydrotalcites." J Catal, 220(1): 161-171.

18. Shi, F. W, Chen ,Y. G. and Sun, L. P. (2012) "Hydroxylation of phenol catalyzed by different forms of $\mathrm{Cu}$-alginate with hydrogen peroxide 
as an oxidant[J]." CatalCommun., 25:102-105.

19. Shi, R. X, Yang, P. and Dong, X. B. (2013) "Growth of flower-like $\mathrm{ZnO}$ on $\mathrm{ZnO}$ nanorod arrays created on zinc substrate through low-temperature hydrothermal synthesis." Appl Surf Sci, 264:162-170.

20. Tatsumi, T, Tajima, H. and Yamamoto, K. (1993) "Epoxidation of Alkenes Catalyzed by Decatungstate as Pillars in Layered Double Hidroxides." Stud Surf Sci Catal, 75: 1703-1706.

21. Timofeeva, M. N, Hasan, Z. and Panchenko, V. N. (2012) "Vanadium-containing nickel phosphate molecular sieves as catalysts for $\alpha$-pinene oxidation with molecular oxygen: A study of the effect of vanadium content on activity and selectivity." J Mol Catal A: Chem, 363:328-334.

22. Tzialla, A. A, Pavlidis, I. V. and Felicissimo, M. P. (2010) "Lipase immobilization on smectite nanoclays: Characterization and application to the epoxidation of $\alpha$-pinene." Bioresour Technol, 101(6):1587-1594.

23. Zhi, Y, Li, Y. G. and Zhang, Q. H. (2010) "ZnO Nanoparticles Immobilized on Flaky Layered Double Hydroxides as Photocatalysts with Enhanced Adsorptivity for Removal of Acid Red G."Langmuir, 26(19): 15546-15553. 